\title{
Al lifecycle models need to be revised
}

\section{An exploratory study in Fintech}

\section{Mark Haakman $^{1} \cdot$ Luís Cruz $^{2}$ (D) $\cdot$ Hennie Huijgens ${ }^{1} \cdot$ Arie van Deursen $^{2}$}

Accepted: 28 May 2021 / Published online: 8 July 2021

(C) The Author(s) 2021

\begin{abstract}
Tech-leading organizations are embracing the forthcoming artificial intelligence revolution. Intelligent systems are replacing and cooperating with traditional software components. Thus, the same development processes and standards in software engineering ought to be complied in artificial intelligence systems. This study aims to understand the processes by which artificial intelligence-based systems are developed and how state-of-the-art lifecycle models fit the current needs of the industry. We conducted an exploratory case study at ING, a global bank with a strong European base. We interviewed 17 people with different roles and from different departments within the organization. We have found that the following stages have been overlooked by previous lifecycle models: data collection, feasibility study, documentation, model monitoring, and model risk assessment. Our work shows that the real challenges of applying Machine Learning go much beyond sophisticated learning algorithms - more focus is needed on the entire lifecycle. In particular, regardless of the existing development tools for Machine Learning, we observe that they are still not meeting the particularities of this field.
\end{abstract}

Keywords AI engineering $\cdot$ AI lifecycle $\cdot$ SE4AI $\cdot$ Machine learning $\cdot$ Case study

\author{
Communicated by: Tim Menzies \\ Luís Cruz \\ 1.cruz@tudelft.nl \\ Mark Haakman \\ Mark.Haakman@ing.com \\ Hennie Huijgens \\ Hennie.Huijgens@ing.com \\ Arie van Deursen \\ Arie.vanDeursen@tudelft.nl
}

1 AI For Fintech Research, ING, Amsterdam, Netherlands

2 Delft University of Technology, Delft, Netherlands 


\section{Introduction}

Artificial Intelligence (AI) has become increasingly important for organizations to support customer value creation, productivity improvement, and insight discovery. Pioneers in the AI industry are asking how to better develop and maintain AI software (Menzies 2019). This paper focuses on Machine Learning, the branch of AI that deals with the automatic generation of knowledge models based on sample data. Throughout this article, Machine Learning and AI are used interchangeably.

Although most of the AI techniques are not so recent (e.g., neural networks were already being applied in the 1980s (Mead and Ismail 1989)), the recent access to large amounts of data and more computing power has exploded the number of scenarios where AI can be applied (Wu et al. 2019; Bernardi et al. 2019). In fact, AI is now being used to add value in critical business scenarios. Consequently, a number of new challenges are emerging in the lifecycle of AI systems, comprising all the stages from their conception to their retirement and disposal. Like normal software applications, these projects need to be planned, tested, debugged, deployed, maintained, and integrated into complex systems.

Companies leading the advent of AI are reinventing their development processes and coming up with new solutions. Thus, there are many lessons to be learned to help other organizations and guide research in a direction that is meaningful to the industry. This is particularly relevant for heavily-regulated industries such as fintech. Industries in the fintech domain ought to make sure that not only they adhere to ever-changing regulations ${ }^{1}$ but also that the usage of their products is compliant. Hence, new processes need to be designed to make sure AI systems meet all required standards.

Recent research has addressed how developing AI systems is different from developing regular Software Engineering systems. A case study at Microsoft identified the following differences (Amershi et al. 2019): 1) data discovery, management, and versioning are more complex; 2) practitioners ought to have a broader set of skills; and 3) modular design is not trivial since AI components can be entangled in complex ways. Unfortunately, existing research offers little insight into the challenges of transforming an existing IT organization into an AI-intensive one.

Examples of existing models describing the Machine Learning lifecycle are the CrossIndustry Standard Process for Data Mining (CRISP-DM) (Shearer 2000) and the Team Data Science Process (TDSP) (Ericson et al. 2017). However, Machine Learning is being used for different problems across many different domains. Given the fast pace of change in AI and recent advancements in Software Engineering, we suspect that there are deficiencies in these lifecycle models when applied to a fintech context.

To remedy this, we set out this exploratory case study aimed at understanding and improving how the fintech industry is currently dealing with the challenges of developing Machine Learning applications at scale. ING is a relevant case to study, since it has a strong focus on financial technology and Software Engineering and it is undergoing a bold digital transformation to embrace AI as an important competitive factor. By studying ING, we provide a snapshot of the rapid evolution of the approach to Machine Learning development.

We define the following research questions for our study:

RQ1:How do existing Machine Learning lifecycle models fit the fintech domain?

\footnotetext{
${ }^{1}$ Bank regulations change every 12 minutes by Chris M. Skinner (2017). Retrieved on July 8, 2021: https:// thefinanser.com/2017/01/bank-regulations-change-every-12-minutes.html/
} 
RQ2: What are the specific challenges of developing Machine Learning applications in fintech organizations?

We interviewed 17 people at ING with different roles and from different departments. Thereafter, we triangulated the resulting data with other resources available inside the organization. Furthermore, we refine the existing lifecycle models CRISP-DM and TDSP based on our observations at ING.

Our results unveil important challenges that ought to be addressed when implementing Machine Learning at scale. Feasibility assessments, documentation, model risk assessment, and model monitoring are stages that have been overlooked by existing lifecycle models. There is a lack of standards and there is a need for automation in the documentation and governance of Machine Learning models. Finally, we pave the way for shaping the education of AI to address the current needs of the industry.

The remainder of this paper is structured as follows. In Section 2 we introduce existing lifecycle models and describe related work. In Section 3, we outline the study design. We report the data collected in Section 4 and we answer the research questions in Section 5. We discuss our findings and threats to validity in Section 6. Finally, in Section 7, we pinpoint conclusions and outline future work.

\section{Background}

In this section, we present the lifecycle models considered in this study and examine existing literature outlining the differences with our study.

\subsection{Existing Lifecycle Models}

In this study, we consider three reference models for the lifecycle of Machine Learning applications: Cross-Industry Standard Process for Data Mining (CRISP-DM) (Shearer 2000), the Team Data Science Process (TDSP) (Ericson et al. 2017), and the Microsoft model described by Amershi et al. (2019). We chose CRISP-DM, as although it is twenty years old, it is still the de facto standard for developing data mining and knowledge discovery projects (Martínez-Plumed et al. 2019). We selected TDSP as modern industry methodology, which has at a high level much in common with CRISP-DM. Finally, we also include the model described by Amershi et al., which is based on CRISP-DM and TDSP and addresses the workflow of software engineering teams (Amershi et al. 2019). There are other methodologies, but most are similar to these three. Findings in our paper can be extrapolated to those other lifecycle models.

CRISP-DM aims to provide anyone with "a complete blueprint for conducting a data mining project" (Shearer 2000). Although data mining is not the common term used nowadays, it is valid for any project applying scientific methods to extracting value from data, including Machine Learning (Martínez-Plumed et al. 2019). CRISP-DM breaks down a project into six phases, as presented in Fig. 1. It typically starts with Business Understanding to determine business objectives, going back and forward with Data Understanding. It is followed by Data Preparation to make data ready for Modeling. The produced model goes through an Evaluation in which it is decided whether the model can go for Deployment or it needs another round of improvement. The arrows between stages indicate the most relevant and recurrent dependencies, while the arrows in the outer circle indicate the evolution of Machine Learning systems after being deployed and their iterative nature. 


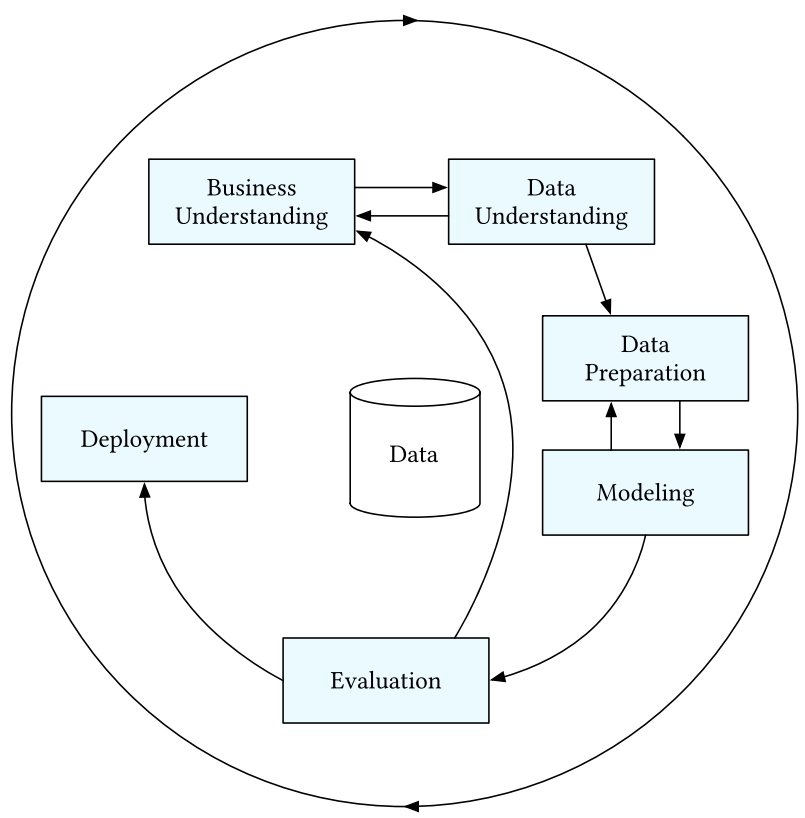

Fig. 1 Cross-industry standard process for data mining (CRISP-DM)

Based on CRISP-DM, a number of lifecycle models have been proposed (MartínezPlumed et al. 2019; Mariscal et al. 2010) to address varying objectives. Derived models extend CRISP-DM for projects with geographically dispersed teams (Moyle and Jorge 2001), with large amounts of data and more focus on automation (Wu et al. 2013; Rollins 2015), or targeting the model reuse across different contexts (Martínez-Plumed et al. 2017).

TDSP is "an agile, iterative data science methodology" proposed by Microsoft, to deliver Machine Learning solutions efficiently (Ericson et al. 2017). The original methodology includes four major stages, as can be seen in Fig. 2: Business Understanding, Data Acquisition, Modeling and Deployment. As depicted by the arrows in the figure, TDSP proposes stronger dependencies but does not enforce a particular order between stages, emphasizing that different stages can be iteratively repeated at almost any time in the project.

Amershi et al. (2019) describe the nine stages followed by software engineering teams at Microsoft who are integrating machine learning into application and platform development. The workflow is presented in Fig. 3, with nine stages: Model Requirements, Data Collection, Data Cleaning, Data Labeling, Feature Engineering, Model Training, Model Evaluation, Model Deployment, and Model Monitoring. The large feedback arrows in the figure depict stages that can be followed by any of their precedent stages. It is the case of Model Evaluation and Model Monitoring. The smaller feedback arrow shows that Model Training and Feature Engineering are typically revisited iteratively.

Despite the number of advancements proposed in previous work, we argue that they do not tackle AI systems that target challenges faced by the fintech industry. Our work pinpoints the changes that needed to be accommodated for AI systems operating under heavy-regulated scenarios and bringing value over pre-existing non-data-driven approaches. 


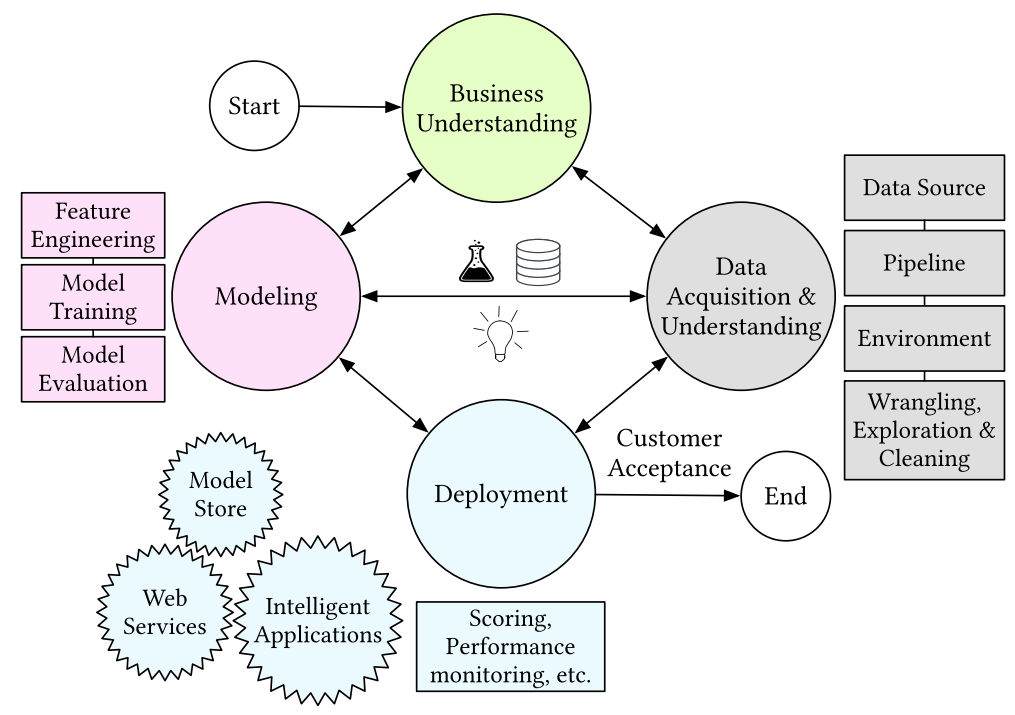

Fig. 2 Team data science process (TDSP)

\subsection{Related Work}

The Machine Learning development lifecycle has been studied in practice in previous research. Amershi et al. (2019) have conducted a case study at Microsoft to study the differences between Software Engineering and Machine Learning. They interviewed 15 software engineers and a conducted a survey with 551 software engineers, yielding 4 main contributions: 1) a description of a machine learning workflow, that we use for comparison with our study; 2) a set of best practices for building applications with machine learning models, 3) a preliminary maturity model for teams developing machine learning applications, and 4) a discussion of the fundamental differences between developing software systems that integrate machine learning models and traditional software systems.

According to Amershi et al. (2019), the main differences that set machine learning apart from traditional software engineering can be summarized as follows: a) handling the data needed for Machine Learning applications is considerably more complex, b) model customization and reuse require a wide set of skills that are not typically found in software teams, c) it is hard to isolate two different machine learning models that operate in the same system - often they ought to be developed and training together. We complement this study by not restricting our observations to software engineering teams. Hence, we include any

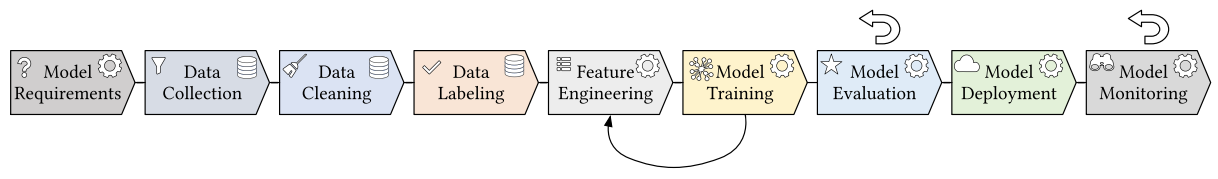

Fig. 3 Microsoft's machine learning workflow described by Amershi et al. (2019) 
practitioner working on a machine learning system. Moreover, we argue that Microsoft as a long history in developing machine learning systems, which might neglect some of the challenges that organizations shifting to AI have to endure. Finally, we compare our observations with existing Machine Learning lifecycle models, including the one proposed by Amershi et al..

Another case study from industry has been performed at Booking.com by Bernardi et al. (2019). In contrast with academic research in which Machine Learning models are validated by means of an error measurement, models at Booking.com are validated through business metrics such as conversion or cancellations. The paper describes process stages such as model designing, deployment, monitoring, and evaluation, but no formal lifecycle model is defined. Moreover, we hypothesize that the fintech domain poses extra challenges that stem from having to adhere to heavy regulations.

Hill et al. (2016) studied how people develop intelligent systems in practice. The study leverages a high-level model of the process and identifies the main challenges. Results show that developers struggle with establishing repeatable processes and that there is a basic mismatch between the tools available versus the practical needs. In this study, we extend the work by Hill et al. by looking more closely at what happens after the Machine Learning model has been evaluated, for example regarding its deployment and monitoring.

The paper by Lin and Ryaboy (2013) describes the big data mining cycle at Twitter, based on the experience of the two authors. The main points made are that for data-driven projects, most time goes to preparatory work before, and engineering work after the actual model training and that a significant amount of tooling and infrastructure is required. In our study, we validate the recommendations of these two experts with a case study with seventeen participants.

Concrete challenges data scientists face are elaborated upon in the study by Kim et al. (2017). They have surveyed 793 professional data scientists at Microsoft. An example of a challenge found is that the proliferation of data science tools makes it harder to reuse work across teams. This challenge is also reinforced in the study by Ahmed et al. (2019). As models are mostly implemented without standard API, input format, or hyperparameter notation, data scientists spend considerable effort on implementing glue code and wrappers around different algorithms and data formats to employ them in their pipelines. Ahmed et al. (2019) show evidence that most models need to be rewritten by a different engineering team for deployment. The root of this challenge lies on runtime constraints, such as a different hardware or software platform, and constraints on the pipeline size or prediction latency.

More studies looked at Machine Learning from a Software Engineering viewpoint. Sculley et al. (2015) identified a number of Machine Learning-specific factors that increase technical debt, such as boundary erosion and hidden feedback loops. Breck et al. (2017) have proposed 28 specific tests for assessing production readiness for Machine Learning applications. These tests include tests for features and data, model development, infrastructure, and monitoring. Arpteg et al. (2018) have identified Software Engineering challenges of building intelligent systems with deep learning components based on seven projects from companies of different types and sizes. These challenges include development, production, and organizational challenges, such as experiment management, dependency management, and effort estimation. In this current study, we will extend this line of research and identify where Software Engineering can help mitigate inefficiencies in the development and evolution of Machine Learning systems. 


\section{Research Design}

To identify the gaps in the existing Machine Learning lifecycle models and explore key challenges in the field, we perform a single-case exploratory case study. This is a recurrent methodology to define new research by looking at concrete situations and to shed empirical light on existing concepts and principles (Yin 2017). We follow the guidelines proposed by Brereton et al. (2008) and Yin (2017) case study methodology.

It is not our objective to build an entirely new theory from the ground up. For that reason, we do not adopt a Grounded Theory (GT) approach, although we do use a number of techniques based on GT (Stol et al. 2016): e.g., theoretical sampling, memoing, memo sorting, and saturation.

The design of the study is further described in this section.

\subsection{The Case}

The case under study is ING, a global bank with a strong European base. ING offers retail and wholesale banking services to 38 million customers in over 40 countries, with over 53,000 employees (ING 2019). ING has a strong focus on fintech, the digital transformation of the financial sector, and professionalization of AI development.

A bank of this size has many use cases where Machine Learning can help. Examples include traditional banking activities such as assessing credit risk, the execution of customer due diligence and transaction monitoring requirements related to fighting financial economic crime. Other examples of use cases are improving customer service and IT infrastructure monitoring.

ING is currently leveraging a major shift in the organization to adopt AI to improve its services and increase business value. As part of it, ING is defining standards for the different processes around the lifecycle of Machine Learning applications. The challenges that ING is facing at the moment make it an interesting case for our study and allow us to identify gaps between current challenges by the industry and academia.

\subsection{Research Methodology}

Semi-structured interviews are the main source of data in this case study. The data is later triangulated with other resources available inside the organization. The approach used to collect information from interviews and to report data is based on the guidelines proposed by Halcomb and Davidson (2006). It is a reflexive, iterative process:

1. Audio taping of the interview and concurrent note-taking.

2. Reflective journaling immediately post-interview.

3. Listening to the audiotape and revising memos.

4. Triangulation.

5. Data analysis.

\subsubsection{Participants}

We selected interviewees based on their role and their involvement in the process of developing Machine Learning applications. We strove to include people of many different roles and from many different departments. The starting position for finding interviewees was the lead of a Software Analytics research team within ING. More interviewees were found 
by the recommendations of other interviewees. The interviewees were also able to suggest other sources of evidence that might be relevant. We increased the number of participants until we reached a level of saturation in the remarks mentioned by interviewees for each stage of the lifecycle.

We adopt a basic approach to assess data saturation. We assume that we achieve data saturation when practitioners from different teams cease bringing insights that we have not observed in previous interviews. Moreover, we only stop collecting data after having data saturation with three consecutive participants.

In total, we interviewed seventeen participants. An overview of the selected participants, with their role and department, can be seen in Table 1. The sixth interview involved two participants. Therefore, they are labeled as P06a and P06b.

\subsubsection{Interview Design}

The first two authors conducted the interviews, which took approximately one hour. We took notes during the interviews and we recorded the interviews with the permission of the participants. An example of the notes taken with P09 is shown in Fig. 4. This section outlines the main steps of our interview design. The full details can be found in our corresponding case study protocol (Haakman et al. 2020).

As interviewers, we started by introducing ourselves and provided a brief description of the purpose of the interview and how it relates to the research being undertaken. We asked the interviewees to introduce themselves and describe their main role within the organization. After the introductions, we asked the interviewee to think about a specific Machine Learning project he or she was working on recently. Based on that project, we

Table 1 Overview of interviewees

\begin{tabular}{lll}
\hline ID & Role & Department \\
\hline P01 & IT Engineer & Application Platforms \\
P02 & IT Engineer & IT Infrastructure Monitoring \\
P03 & Productmanager & Financial Crime \\
P04 & IT Architect & Enterprise Architects \\
P05 & IT Engineer & IT4IT \\
P06a* & Advice Professional & Model Risk Management \\
P06b* & Advice Professional & Model Risk Management \\
P07 & Manager IT & Global Engineering Platform \\
P08 & Feature Engineer & Data \& Analytics \\
P09 & Data Scientist & Wholesale Banking Analytics \\
P10 & Data Scientist & Chapter Data Scientists \\
P11 & IT Engineer & Application Platform \\
P12 & Data Scientist & AIOps \\
P13 & Data Scientist & Wholesale Banking Analytics \\
P14 & Data Scientist & Financial Crime \\
P15 & Data Scientist & Analytics \\
P16 & Data Scientist & Chapter Data Scientists \\
\hline
\end{tabular}

\footnotetext{
* The sixth interview involved two participants, labeled P06a and P06b
} 


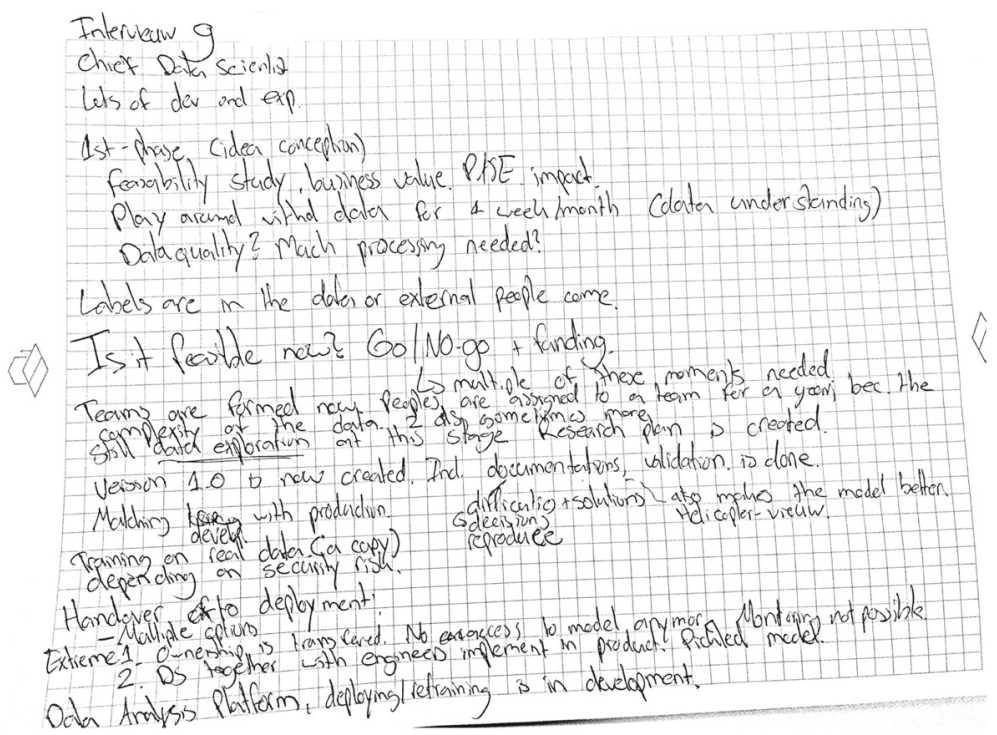

Fig. 4 Excerpt of the notes taken during an interview

asked the interviewee to describe all the different stages of the project. In particular, we asked questions to understand the main challenges they faced and the solutions they had to design.

\subsubsection{Post-interview Strategy}

Right after each interview, the two interviewers got together for a collaborative memoing process (also called reflective journaling (Halcomb and Davidson 2006)) combined with thematic coding. Memoing is the review and formalization of field-notes and expansion of initial impressions of the interaction with more considered comments and perceptions. Memoing is chosen over creating verbatim transcriptions, because the costs associated with interview transcription, in terms of time, physical, and human resources, are significant. An example of the output of memoing is depicted in Fig. 5. Also, the process of memoing assisted the researchers to capture their thoughts and interpretations of the interview data (Wengraf 2001). The audio recordings could still be used to facilitate a review of the interviewers' performance, and assist interviewers to fill in blank spaces in their field notes and check the relationship between the notes and the actual responses (Fasick 1977).

The interviewers took between 30-45 minutes to refine their notes. In this process, the notes were revised and coded into themes based on the particular lifecycle stage that it addressed. We resort to the thematic analysis technique (Fereday and Muir-Cochrane 2006) to derive themes. Thematic analysis is a qualitative data analysis method divided into four steps: 1) familiarization with data, 2) generating initial labels, 3) reviewing themes, 4) defining and naming themes. This technique has been successfully used in previous software engineering studies to extract patterns from software (Cruz and Abreu 2019).

The first two authors worked together to discuss and validate the themes. After the first iteration of label generation - step 2 of thematic analysis - we counted with 49 labels. An example of the derived labels is depicted in Fig. 5. This step was followed by reviewing 


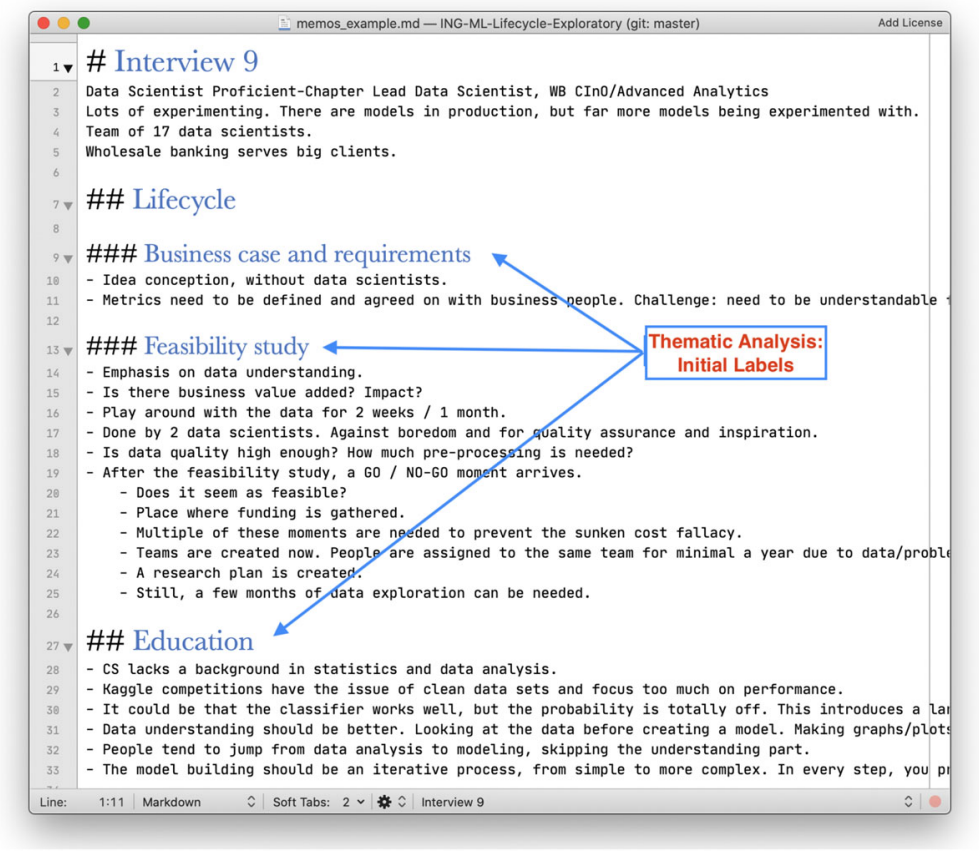

Fig. 5 Excerpt of the results of the memoing process. The notes were assigned under different lifecycle themes

themes - step 3 of thematic analysis - in which we discussed each label and looked for other labels that could be redundant. For example, we merged the labels Feasibility Study and Proof of Concept together into a single theme. This step yielded 11 overarching themes that we further detail in Section 4.

Whenever possible, the selected themes follow the nomenclature of existing frameworks - namely, CRISP-DM, TDSP, and Amershi et al.'s model. Alternative themes were created when 1) we encountered notes that did not fit the existing themes, or 2) there was a theme being mentioned on different occasions which helped understand a particular part of the process.

After some time, the interviewers amended the memos by reviewing the audiotapes. The purpose of this stage was to ensure that the memos provided an accurate reflection of the interviews (Halcomb and Davidson 2006). Each interview resulted in three artifacts: the recording of the interview, the field notes taken during the interview, and the memos as a result of the above-mentioned memoing.

\subsubsection{Triangulation}

The main goal of triangulation is to provide means of assessing the validity of insights from practitioners. We used the documentation in the intranet of ING to gain a deeper understanding of the platforms and processes mentioned in the interviews. This documentation is available to all employees in the organization and aims to provide a clear understanding of the processes and resources available. It typically consists of slide decks, short guides, 
and webpages. This documentation is confidential, thus triangulation was performed by the authors affiliated at ING.

Ultimately, triangulation did not serve as a mean to discard insights, but rather to understand their relevance and whether they generalize to other sectors of the organization. As an example, we observed that, although several teams mentioned being using templates to document machine learning projects, these are not available to the rest of the organization. On contrary, we have analyzed several resources regarding feasibility studies - meaning that this is a well-established standard at ING.

\section{Data Analysis}

The input of the interviewees does not answer the research questions directly. Therefore, we report the resulting data of the interviews in this section and we use this data to answer the research questions later in Section 5.

We organize the data among eight core Machine Learning lifecycle themes: problem design, requirements, data engineering, modeling, documentation, model evaluation, model deployment, and model monitoring. Overarching data that does not fit a single lifecycle stage is categorized under testing, iterative development, and education. In some cases, subthemes were also defined: Feasibility study, Model Risk Assessment, Data Collection, Data Understanding, and Data Preparation. These stages were determined based on the thematic coding described in Section 3.2.3. We refrain from describing details that did not add to existing lifecycle models (e.g., model training).

For all the remarks, we identify the practitioner who mentioned them by referencing the corresponding ID from Table 1. Given that this is a qualitative analysis, the number of individuals supporting a particular result has no quantitative meaning on its relevance. The end of each category provides a highlight box with a summary of the main results.

\subsection{Problem Design}

Machine Learning projects at ING start with the definition of the problem that needs to be solved. Two main approaches are observed in this study:

1. Innovation push: a stakeholder comes up with a question or problem that needs to be solved. A team is set up to design a solution using a suitable Machine Learning technique.

2. Technology push: a team identifies new data or a set of Machine Learning techniques that may add business value and are potentially useful or solving problems within the organization. This approach aims to optimize processes, reduce manual work, increase model performance, and create new business opportunities.

The problem is defined together with stakeholders and it is assessed whether using Machine Learning is appropriate to solve the problem (P01, P14, P15). In the teams of P15 and P14, this is done by collaboratively filling in a project document with the stakeholders which contains information like the problem statement, goals, and the corresponding business case. Also, domain experts outside the teams are part of this.

Machine Learning projects start with a problem statement which is used to discuss whether a Machine Learning solution is necessary. This step requires high engagement from problem domain experts. 


\subsection{Requirements}

Besides project-specific requirements, many of the requirements come from the organization and are applicable to every Machine Learning application (P15). These requirements include traceability, interpretability, and explainability (P01, P04, P07, P15). Together with all other regulatory requirements, they pose a big challenge while developing Machine Learning applications (P04). A natural consequence of regulatory requirements is that black-box AI models cannot be used in most situations (P01, P04, P14). For risk management safeness, only interpretable/explainable AI models are accepted.

Project-specific requirements are often defined by the product owner together with the stakeholders (P10). Data requirements are said to become more clear while working with the model (P04). As the users of the system are often no Machine Learning experts, defining the model performance requirements is sometimes a challenge (P09, P13).

Requirements are not always defined beforehand. Data and Model requirements become more clear while working with an initial model. Requirements related to traceability, interpretability, and explainability are typically defined a the organizational level.

\subsection{Data Engineering}

Interviewees describe that data engineering requires the major part of the lifetime of a Machine Learning project (P03, P10, P15) and is also the most important for the success of the project (P10).

\subsubsection{Data Collection}

Data collection is considered a very challenging and time-consuming task (P03, P04, P12, P14). Typical use cases require access to sensitive data, which needs to be formally requested. ING has an extensive data governance framework that, among others, assigns data management roles (e.g. data owner) and rules for obtaining, sharing, and using data. Each dataset is assigned a criticality rating, to define the degree of data governance and control required.

There might be people with different access privileges to data in the same project. This means that, in the exploratory stages of some projects using critical data, only a restricted number of team members (e.g., data scientists) are able to perform an exploratory analysis of data. The remaining practitioners will only have access to the model specification (P04).

A challenge of data collection is making sure that the (training and test) data collected is representative of the problem (P13). As an example, if a Machine Learning model is trained on systems logs, it should be made sure that logs of all systems are available. Another challenge is merging data from multiple sources (P10, P12). Going back to the logging example, different systems may have different logging formats, but the configurations of these formats cannot be altered by the developers creating the model.

\subsubsection{Data Understanding}

In the data understanding stage, an assessment is done on the quality of available data and how much processing will be required to use that data. It comprises exploratory data analysis, often including graphical visualizations and summarization of data. According to P09, 
the temptation of applying groundbreaking Machine Learning techniques tends to overlook the importance of understanding the data.

Data understanding is also an important step to assess the feasibility of the project. Thus, it entails not only performing an exploratory analysis, but also a considerable effort in communicating the main findings to all the different stakeholders. This tends to be a slow process (P12).

\subsubsection{Data Preparation}

After the data is collected and it is assessed that the data is representative of the problem being solved, the data is prepared to be used for modeling.

A challenge regarding data preparation is that the same pre-processing has to be ensured in the development environment and in the production environment (P08, P09). Data streams in production are different than in the development environment and it is easier to clean training and testing data than production data (P09).

Collecting, understanding, and preparing data are the most time-consuming stages of Machine Learning projects. There is a meticulous data access control that, despite being quintessential, sets major obstacles in understanding the data and performing exploratory analyses. Practitioners emphasize, data understanding implies being able to communicate it to other stakeholders. Finally, the differences between development and production environments pose challenges for data preparation.

\subsection{Modeling}

Model training is mostly done in on-premises environments such as Hadoop ${ }^{2}$ and Spark $^{3}$ clusters (P09) or in generic systems using, for example, the scikit-learn ${ }^{4}$ library (P01). These private platforms are connected with the data lakes where data is stored, so training can be done on (a copy of) real production data (P01, P03). The on-premises environment has no outgoing connection to the internet, so a connection to other cloud services such as Microsoft Azure ${ }^{5}$ or Google Cloud ${ }^{6}$ is not possible (P08). This means that data scientists are limited to the tools and platforms available within the organization when dealing with sensitive data. Also, all project dependencies need to be previously approved, after which they are made available in a private package repository (P04, P12), which contains whitelisted packages that have been internally audited. This can be frustrating, when new ground-breaking AI technologies appear, practitioners have to wait before they can explore the potential of those technologies at ING (P12) - we later refer to this challenge as Technology Access (cf. Section 5). Fewer restrictions are in place if Machine Learning is applied to public data, for example on stock prices. In that case, external cloud services and packages may be used (P09).

\footnotetext{
${ }^{2}$ Hadoop enables distributed processing of large data sets across clusters of computers https://hadoop.apache. org

${ }^{3}$ Spark is a unified analytics engine for large-scale data processing. https://spark.apache.org

${ }^{4}$ Scikit-learn is a Machine Learning library for Python. https://scikit-learn.org

${ }^{5}$ Microsoft Azure is a cloud computing service. https://azure.microsoft.com/en-us

${ }^{6}$ Google Cloud is a cloud computing service. https://cloud.google.com
} 
Model training is an iterative process. Usually, multiple models are created for the same problem. First, a simple model is created (e.g., a linear regression model) to set as a baseline (P09). In the following iterations, more advanced models are compared to this baseline model. If an approach other than Machine Learning already exists (e.g., rule-based software), the models are also compared with this.

To keep track of different versions of models, different teams use different strategies. For example, the team of P08 keeps track of an experiment log using a spreadsheet, in which the training set, validation set, model, and pre-processing steps are specified for each version. This approach for versioning is preferred over solutions like MLFlow ${ }^{7}$ for the sake of simplicity (P08, P15).

\subsubsection{Model Scoring}

An implicit sub stage of modeling is assessing model performance to measure how well the predictions of the model represent ground truth data.

We define Model Scoring as assessing the performance of the model based on scoring metrics (e.g., f1-score for supervised learning). It is also known as Validation by the Machine Learning community, which should not be confused with the definition by the Software Engineering community ${ }^{8}$ (Ryan and Wheatcraft 2017; 15288 2015).

The main remarks for this stage are related to defining the right set of metrics ( $\mathrm{P} 03, \mathrm{P} 06$, $\mathrm{P} 12$, P14, P15, P16). The problem is two-fold: 1) identify the right metrics and 2) communicate why the selected metrics are right. Practitioners report that this is very problem-specific. Thus, it requires a good understanding of the business, data, and learning algorithms being used. From an organization's point of view, these different perspectives are a big barrier to defining validation standards.

The challenges in Modeling summarize as follows: 1) the latest Machine Learning technologies are not always eligible for use; 2) baseline models are essential artifacts for model development; 3) teams keep track of all experiments, which often revolves around keeping a customized spreadsheet; and 4) defining performance metrics is problem-specific, posing a challenge to the definition of standards at the organizational level.

\subsection{Documentation}

Each model has to be documented (P02). This serves multiple goals. It makes assessing the model from a regulatory perspective possible ( $\mathrm{P} 09, \mathrm{P} 13)$, it enables reproducibility, and also can make the model better because it is looked at from a broad perspective - i.e., a "helicopter view" (P09). It also provides an audit trail of actions, decisions, versions, etc. that supports evidencing. Documentation also supports the transfer of knowledge, for example, to new team members or the end-users which are mostly not Machine Learning experts (P12). Just like code, documentation is also peer-reviewed (P13).

\footnotetext{
${ }^{7}$ MLFlow is a platform to manage the Machine Learning lifecycle. https://mlflow.org

${ }^{8}$ Validation in Software Engineering "is the set of activities ensuring and gaining confidence that a system is able to accomplish its intended use, goals and objectives" (15288 2015).
} 
The content of the documentation differs slightly per department, but all documentation should at least follow the minimum standards defined by the model risk management framework (P06). Some teams extend on this by creating templates for documentation themselves (P13). In general, the following is documented when developing a Machine Learning application: the purpose, methodology, assumptions, limitations, and the use of the model. More concretely, a Technical Model Document is created which includes the model methodology, input, output, performance metrics and measurements, and testing strategy (P14). It furthermore states all faced difficulties and their solutions, plus the main (technical) decisions (P09). It has to explain why a certain model is chosen and what its inner workings are, to be able to demonstrate the application does what the creators claim it is doing. Creating documentation is considered overly time-consuming, although necessary (P07).

Documentation is a first-class artifact for regulatory compliance, knowledge transfer, and reproducibility. Hence, a peer-review process is in place to ensure documentation quality.

\subsection{Model Evaluation}

An essential step in the evaluation of the model is communicating how well the model performs according to the defined metrics. It is about demonstrating that the model meets business and regulatory needs and assessing the design of the model. One key difference between the metrics used in this step and the metrics used for Model Scoring is that these metrics are communicated to different stakeholders that do not necessarily have a Machine Learning or data science background. Thus, the set of metrics needs to be extended to a general audience. One complementary strategy used by practitioners is having live demos of the model with business stakeholders (P03, P15, P16). These demos allow stakeholders to try out different inputs and try corner cases.

\subsubsection{Model Risk Assessment}

An important aspect of evaluating a model at ING is making sure it complies with regulations, ethics, and organizational values (P15, P06). This is a common task for any type of model built within the organization - i.e., not only Machine Learning models but also economic models, statistical forecasting models, and so on. In the interviews, Model Risk Assessment was mentioned as mandatory within the model governance strategy, undertaken in collaboration with an independent specialized team (P06, P14). This is a long-stablished stage which is now being challenged by the specifics of Machine Learning. For example, traditional risk assessment teams did not initially have the right Machine Learning expertise to evaluate the models with confidence.

Depending on the criticality level of the model, the intensity of the review may vary. Each model owner is responsible for the risk management of their model, but colleagues from the risk department help and challenge the model owner in this process.

During the periodic risk assessment process, assessors inspect the documentation provided by the Machine Learning team to assess whether all regulations and minimum standards are followed. The documentation used in this stage is considered to be overly time-consuming, as emphasized by $\mathrm{P} 07$ : "70\% percent of the time people are writing Word documents to explain their code is compliant.”. Although the process is still under development within ING, the following key points are being covered (P06): 1) model identification 
(identify if the candidate is a model which needs risk management), 2) model boundaries (define which components are part of the model), 3) model categorization (categorize the model into the group of models with a comparable nature, e.g. anti-money-laundering), 4) model classification (classify the model into in the class of models which require a comparable level of model risk management), and 5) assess the model by a number of sources of risk.

Although Model Risk Assessment is not new to the fintech industry, Machine Learning is requiring a revised approach. Currently, developers endure considerable efforts to create the required documentation.

\subsection{Model Deployment}

We observed three deployment patterns at ING:

1. A specialized team creates a prototype with a validated methodology, and an engineering team takes care of reimplementing it in a scalable, ready-to-deploy fashion. In some cases, this is a necessity due to the technical requirements of the model, e.g., when models are developed in Python, but should be deployed in Java (P08, P09, P13).

2. A specialized team creates a model and exports its configuration (e.g., a pickle ${ }^{9}$ and required dependencies) to a system that will semi-automatically bundle it and deploy it without changing the model (P01, P09).

3. The same team takes care of creating the model and taking it into production. This mostly means that software engineers are part of the team and a structured and strict software architecture is ensured.

Similar to the training environments, Machine Learning systems are deployed to onpremises environments. A reported challenge regarding the deployment environment is that different hardware and platform parameters (e.g., Spark parameters) can result in different model behavior or errors (P16). For example, the deployment environment may have less memory than the training environment. Furthermore, the resources for a Machine Learning system are dynamically allocated whenever needed. However, it is not trivial understanding when a system is no longer needed and should be scaled down to zero (P01).

There are deployment patterns in which a separate team needs to reimplement the model to meet production settings.

\subsection{Model Monitoring}

After having a model in production, it is necessary to keep track of its behavior to make sure it operates as expected. It implies testing the model while the model is deployed online. The main advantage is that it uses real data. Previous work refers to this stage as online testing (Zhang et al. 2020).

\footnotetext{
${ }^{9} \mathrm{~A}$ pickle is a serialized Python object. https://docs.python.org/3/library/pickle.html
} 
The inputs and outputs of the model are monitored while it is executing. Each model requires a different approach and different metrics, as standards are not yet defined. In this stage, practitioners also look into whether the statistical properties of the target variable do not change in unforeseen ways (P11). The model behavior is mostly monitored by data science teams and is still lacking automation (P03, P05, P06, P14). Also, the impact on user experience is monitored when the model has a direct impact on users. This is mostly done using A/B testing techniques and can have business stakeholders directly involved (P03, P10).

Teams resort to self-developed or highly-customized dashboard platforms to monitor the models (P15, P16). Within the organization, different teams may have different platforms. While standardization is in development, for now, we have not observed solutions that are used across the organization. A big challenge in making these platforms available is the fact that each problem has different monitoring requirements and considerable engineering efforts need to be undertaken to effectively monitor a given model and implement access privileges (P15).

More automation is needed for model monitoring. Teams have created their own automation tools, but making them available to other teams requires unfeasible efforts that do not meet their priorities.

\subsection{Testing}

Testing is a task that is transversal to the whole development process. It is done at the model level and at the software level.

Testing at the model level addresses requirements such as correctness, security, fairness, and interpretability. With the exception of correctness, we have not observed automated approaches to verify these requirements. A challenge for the correctness tests is defining the number of errors that are acceptable - i.e., the right threshold (P14).

For testing at the software level, unit and integration testing is the general approach. It scopes any software used in the lifecycle of the model (P07). It enables the verification of whether the techniques adopted in the design of the Machine Learning system are working as expected. However, although unit and integration testing is part of the checklist used for Model Evaluation, a number of projects are yet not doing it systematically (P12, P15). As reported by P14, tests are not always part of the skill set of a data scientist. Nevertheless, there is a generalized interest in learning code testing best practices (P12).

Although practitioners are eager to learn automated testing practices, this is not part of their skillset. Hence, projects are struggling to adopt unit and integration testing strategies.

\subsection{Iterative Development}

At ING, teams adopt agile methodologies. Three practitioners (P03, P09, P14) mentioned that using agile methodologies is not straightforward in the early phases of Machine Learning projects. They argued that performing a feasibility study does not fit in small iterations. The first sprint requires spending a considerable amount of time understanding and preparing data before being able to deliver any model. On the other hand, 
interviewees acknowledge the benefits of using agile (P03, P14). It helps keep the team focused on practical achievements and goals. Another advantage is that stakeholders are kept in the loop (P14).

Typically, 2-3 data scientists are working together on the same model. For this reason, issues with having many developers working on the same model and merging different versions of a model have not been disruptive yet.

\subsubsection{Feasibility Study}

The end of the first iteration is also a decisive step in the project. Based on the outcome of this iteration there is a go/no-go assessment with all the stakeholders, in which the project is evaluated in terms of viability (i.e., does it solve a business issue), desirability (i.e., is it complying with ethics or governance rules), and feasibility (i.e., cost-effectiveness) (P04, P09, $\mathrm{P} 15, \mathrm{P} 16)$. This process is well-defined within the organization for all innovation projects. According to P04 and P09, feasibility assessments are essential at any point of the project it is important to adopt a fail-fast approach.

All projects must go over a feasibility study in their early stages. Until then, projects do not fit the typical sprint-based agile planning. An agile approach helps practitioners prioritize tasks and engage stakeholders.

\subsection{Education}

Interviewees indicated multiple ways in which education can be improved to make graduates better Machine Learning practitioners in the industry. Firstly, data scientists should have more knowledge of Software Engineering and vice-versa (P01, P11, P14, P16). P11 indicates that data scientists with little software engineering knowledge will produce code that is harder to maintain and likely increases technical debt. On the other hand, a software engineer without data science expertise may write clean code, which nevertheless may not add much business value, because of ineffective data exploration strategies (P09).

Another remark by practitioners is that education should focus more on the process of developing Machine Learning, rather than teaching learning techniques (P08). While graduates are appreciated for their broad sense of the state-of-the-art, they must learn how to tackle Machine Learning issues in large organizations (P08, P10). Academia knows well how to work with new projects, but in reality, the history of the company affects how to perform Machine Learning - e.g., integration with legacy systems (P08). Graduates seem to underestimate the efforts needed for data engineering, especially data collection (P03, P09, P12). Also, too much attention lies solely on the performance of models. In reality, over-complex models cannot be applied in organizations, because they tend to be too slow or too hard to explain (P16). These models - squeezing every bit of performance - are great for data science competitions as facilitated on Kaggle, but not for the industry, where more efficient solutions are necessary (P09, P16).

There is practical value on having a strong background on both Software Engineering and Data Science. Education should put more focus on the process instead of model-training techniques. 


\section{Data Synthesis}

In this section, we answer each research question.

RQ1:How do existing Machine Learning lifecycle models fit the fintech domain?

Our interviews show evidence that existing models do not fit the needs of the today's fintech industry and changes ought to be made.

To explain this further, we pinpoint the differences between lifecycle models existing in the literature and the findings observed in our study. We select three reference models, as described in Section 2.1: CRISP-DM (Shearer 2000), TDSP (Ericson et al. 2017), and Amershi et al. (2019). We justify and define each required change and discuss the constraints to which they generalize outside the case of ING: to the fintech domain or to general Machine Learning projects.

We propose the changes of CRISP-DM in Fig. 6 - new stages are highlighted with orange background and bold text. We add three new essential stages: Data Collection (as part of Data Engineering), Documentation, and Model Monitoring. Furthermore, we emphasize the feasibility assessment with the "Go/No-go" checkpoint and a sub-stage Model Risk Assessment, part of Evaluation.

There are, however, stages identified at ING that naturally fit CRISP-DM. Similarities between CRISP-DM and the stages observed are Business Understanding, Data Understanding, Data Preparation, Modeling, Evaluation, Deployment.

As depicted in Fig. 7, we adapt the TDSP model to include Documentation, Model Evaluation, and Model Monitoring as major stages. We also emphasize Model Risk Assessment (as part of Evaluation) and a Feasibility Study.

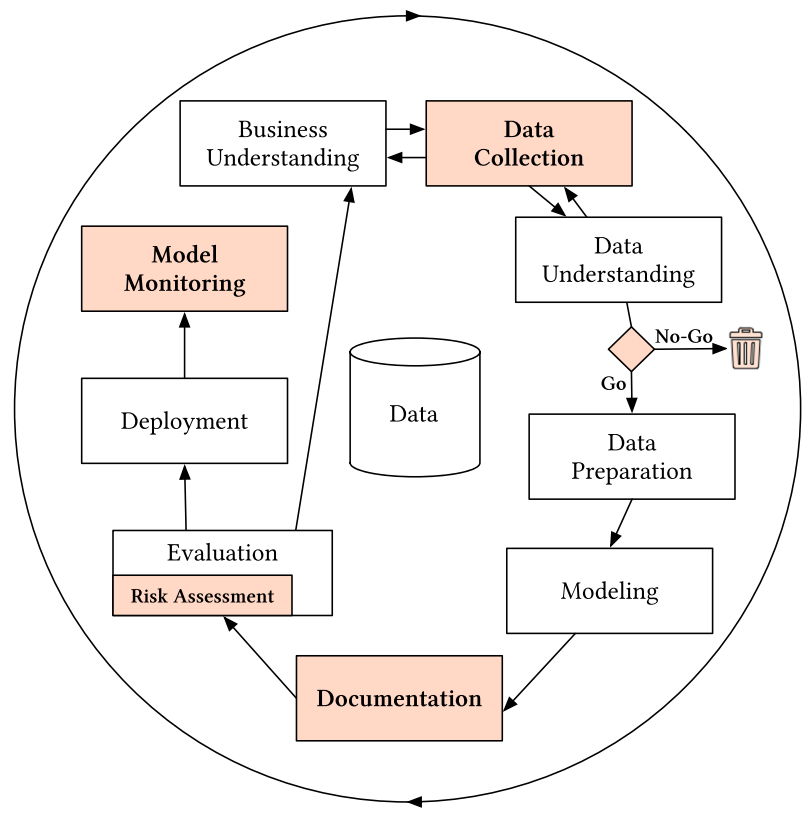

Fig. 6 Refined CRISP-DM model. Additions in red, with bold text 


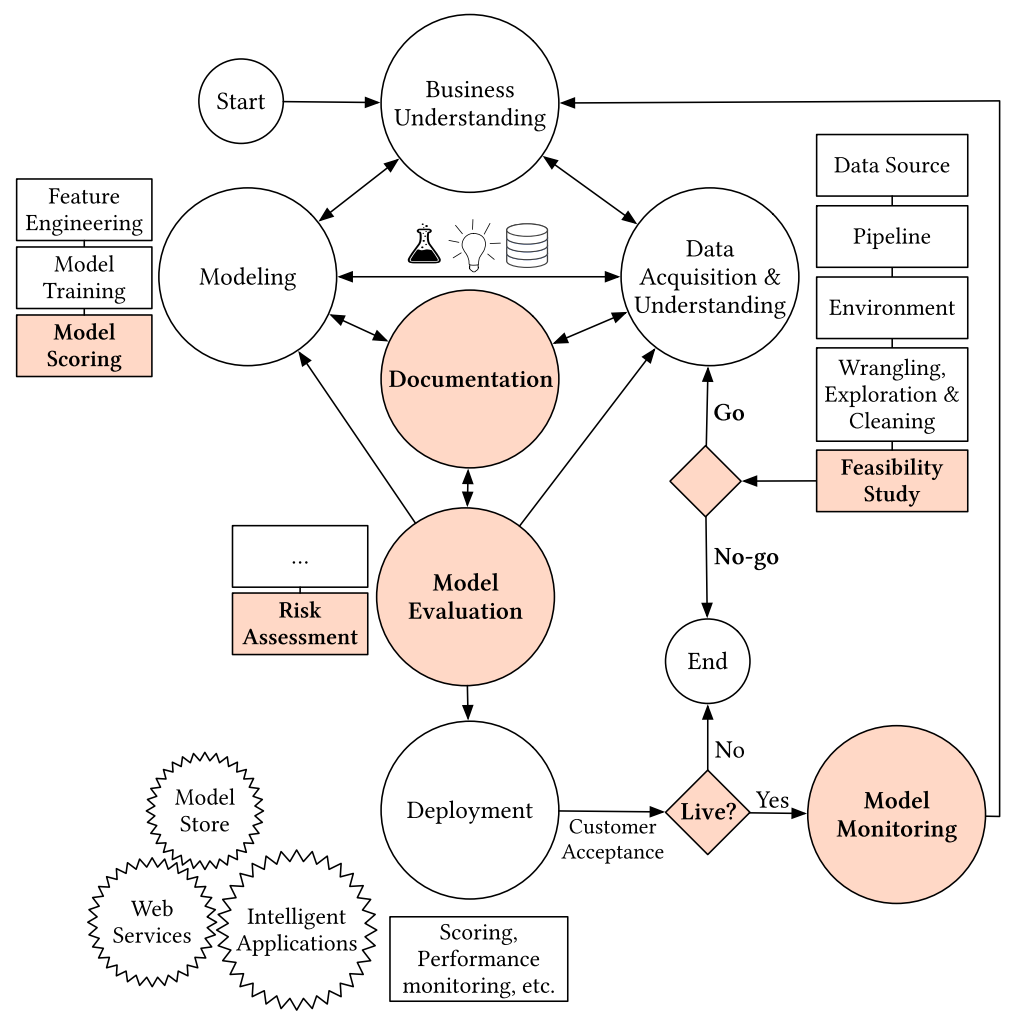

Fig. 7 Refined TDSP model. Additions in red, with bold text

We observed stages that are already being covered by TDSP: Business Understanding, Data Acquisition \& Understanding, Modeling, and Deployment.

After inspecting the model by Amershi et al. (2019), we propose the changes in Fig. 8. We adapt the original model to include Feasibility Study, and Peer-reviewed Documentation. We also emphasize Model Scoring (as part of Model Training) and Risk Assessment (as part of Model Evaluation). Other observations in our study naturally fit the stages described by Amershi et al. (2019): Model Requirements, Data Collection, Data Cleaning, Data Labeling, Feature Engineering, Model Training, Model Evaluation, Model Deployment, Model Monitoring.

There are, however, stages identified at ING that naturally fit CRISP-DM and TDSP. Similarities between TDSP are Business Understanding, Data Acquisition \& Understanding, Modeling, and Deployment.

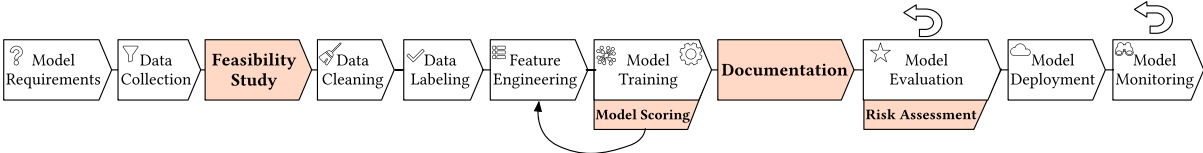

Fig. 8 Refinements to the Microsoft model described by Amershi et al. (2019). Additions in red, with bold text 
The adaptations of the models will be further elaborated upon in the following paragraphs.

Data Collection Although CRISP-DM encompasses Data Collection within Data Understanding and Data Preparation, our observations reveal important tasks and challenges that need to be highlighted. As reported in Section 4.3.1, Data Collection requires getting privileges to access data with different criticality-levels and making sure the data is representative of the problem being tackled. Our proposition is that the characteristics observed at ING regarding this phase generalize to any large organization dealing with sensitive data. TDSP and Amershi et al. already contemplate this stage.

Go/No-go or Feasibility Study The aforementioned Feasibility study (cf. Section 4.10) is an essential part of a Machine Learning project to ensure projects have everything in place to deliver the long-term expectations. It was a recurrent step observed in our study, which is aligned with the agile approach, Fail Fast, promoted at ING and many organizations alike. It may generalize to other cases, depending on the agile culture of the organization.

Documentation In our case, documentation revealed to be a quintessential artifact for a Machine Learning project. Documentation is the key source of knowledge on how the model is designed, evaluated, tested, deployed, and so on. The documentation is used to evaluate, maintain, debug, and keep track of any other decision regarding the model. It is hard to replace documentation with other strategies because stakeholders with a non-technical background also need to understand the model and have confidence in how the Machine Learning model is designed. Although documentation is also important in traditional Software Engineering applications, the codebase is usually the main target of analysis from audits. In Machine Learning, documentation contains important problem-specific decisions that cannot be understood in the code itself. Hence, at ING, Machine Learning practitioners devote a big part of their time to write clear documentation. Furthermore, documentation endures a peer-review process to make sure it is sound and complete. We have no evidence on how this stage generalizes to other organizations, but believe this to be crucial in any highly regulated environment.

Model Evaluation Although the original version of TDSP also included Model Evaluation, it was proposed as an activity under the Modeling stage. The same applies to the model by Amershi et al. that describes Model Evaluation as a stage where "the engineers evaluate the output model on tested or safeguard datasets using pre-defined metric". For this particular purpose, we use the term Model Scoring and consider it a part of the Modeling or Model Training activities. However, there is an important part of the evaluation that requires more stable versions of the models. Moreover, it is undertaken with stakeholders that are not part of the Modeling loop - e.g., live demos with business managers (cf. Section 4.6) - and requires assessing the model in terms of business metrics. Thus, we highlight this part of the evaluation as its own stage. An important part of this stage is model risk assessment, which we further explain below.

Model Risk Assessment Model Risk Assessment is crucial to any banking or finance organization. Although companies in the finance domain already have a big history of traditional risk management, it does not consider Machine Learning models. Moreover, risk assessment teams do not necessarily have a Machine Learning background to make an informed decision. This does not mean that Machine Learning models face a less meticulous risk 
assessment - it rather means that the process will take even more time, being a bottleneck in the lifecycle. Thus, at ING, model risk assessment is enduring a major transformation to make sure this is not a bottleneck in the process. Notwithstanding its importance, none of the studied lifecycle models contemplate this stage.

Model Monitoring Most Machine Learning models operate continuously and produce outputs online. Our study shows that the natural step after deployment is Monitoring - for example, using dashboards - to ensure the model is behaving as expected. Model Monitoring is not explicit in neither CRISP-DM nor TDSP, but it is relevant to any domain. In fact, Amershi et al. already contemplates this stage.

Finally, although not depicted in the proposed lifecycles, Education is a stage implicit throughout the whole lifecycle. We observe that universities and courses on Machine Learning need to provide a more holistic approach to focus on all the different stages of the lifecycle of a Machine Learning system.

A lifecycle stage that we did not yet observe is the end of life of a Machine Learning system - i.e., the Disposal stage. We presume that a disposal stage is not relevant yet due to the recency of Machine Learning in fintech.

RQ2: What are the specific challenges of developing Machine Learning applications in fintech organizations?

We highlighted many challenges of developing Machine Learning applications in Section 4. While most challenges potentially affect any tech-company leading an AIpowered digital transformation, there are two that stand out in the fintech domain: Model Governance and Technology Access.

Model Governance is on top of the agenda of the case in this study. A well-defined process is in place to validate regulations, ethics, and social responsibility in every Machine Learning model. The relevance of this problem to fintech organizations goes beyond Machine Learning applications: math-based financial models have long been deployed under well-defined risk management processes.

Nevertheless, we observe a need to revise and recreate model governance that suits the particularities of models that are now automatically trained. E.g., continuous training - a practice that is essential for any high maturity Machine Learning process (Akkiraju et al. 2020; Lwakatare et al. 2020) - does not fit the traditional model risk assessment approach in fintech. A new set of documentation, and a manual audit are the bare minimum to release a new version of the model. Hence, automated tools for model governance are essential to ensure Machine Learning models comply with regulations and reduce bottlenecks in the development process.

Our results imply that RegTech - the branch of fintech for managing regulatory requirements - is an emergent field with direct contributions to intelligent systems in fintech. Having automated mechanisms to check model compliance with regulations is essential for the adoption of continuous integration for AI systems in fintech companies. This is an important challenge since, according to previous work (Serban et al. 2020), continuous integration is perceived by practitioners as one of the unexploited practices with the most potential.

Moreover, model risk experts are now required to have a strong background in two disjoints fields: 1) Governance, Risk Management, and Compliance and 2) AI. We conjecture 
that this challenge generalizes to other heavy-regulated domains, such as LegalTech and Healthcare Technology.

Technology Access All AI technologies, tools, and libraries need to be audited to make sure they are safe to be used in fintech applications. Only then, practitioners are able to design their Machine Learning systems around the latest technology. This is a challenge that needs to tackled by any organization akin to ING. As presented in Section 4.4, this process can be limiting since new AI technologies are appearing every day. Practitioners willing to try the latest AI technology may feel less motivated since it may take some time before they are approved. As referred in Section 4.1, many problems at ING are triggered by the Technology push. Hence, new business opportunities might be missed if practitioners are not able to experiment the latest AI technologies.

We do not know to what extent Technology Access is also a challenge to software organizations operating in other domains. Previous work suggests that only $8 \%$ of software developers consider an organization's culture and policies highly-influential when selecting third-party software libraries (Larios Vargas et al. 2020). On contrary, 52\% consider it as a low/moderate influential factor. Nevertheless, we argue that similar obstacles might be observed in many other organizations with high-maturity software development processes. Hence, industries that want to shift towards AI-based systems need to be able to quickly, yet safely, adopt new technologies.

\section{Discussion}

In this section, we discuss the implications of our results and elaborate on the threats to the validity of our findings.

\subsection{Implications}

We see the following implications of our results for the fintech industry and for research.

\subsubsection{Implications for Machine Learning Practitioners}

Machine Learning practitioners have to be aware of extra steps and challenges in their process of developing Machine Learning applications. Although not mentioned in existing lifecycle models, the undertaking of feasibility assessments, documentation, and model monitoring, are crucial while developing Machine Learning applications.

\subsubsection{Implications for Process Architects}

Existing lifecycle models provide a canonical overview of the multiple stages in the lifecycle of a Machine Learning application. However, when being applied to a particular context, such as fintech, these models need to be adapted. From our findings, we suspect that this is also the case for other fields where AI is getting increasing importance. Process architects for intelligent systems for healthcare, autonomous driving, among many others, need to look at their lifecycle models from a critical perspective and update the models accordingly.

Moreover, process architects ought to keep an eye on the Technology Access blocker. Our work suggests that the process of approving the latest AI technologies should be ahead of the needs of practitioners. We argue that a pro-active process should be in place to audit AI 
technologies. It is a key factor to explore new business opportunities and to keep developers motivated.

\subsubsection{Implications for Researchers}

Researchers could focus on solving the reported challenges in the Machine Learning lifecycle with additional tool support and reveal challenges of the ML lifecycle in other domains by extending the case study to more organizations and different types of industries.

More automation is required for exploratory data analysis and data integration techniques (Mitchell et al. 2019; Damiani and Frati 2018). Moreover, there are minimal advancements in documentation of Machine Learning projects. Techniques ought to be studied to help trace documentation back to the codebase and vice versa.

Furthermore, solutions to challenges in the ML lifecycle should be researched. Our study shows that, despite the increasing trend on improving the state-of-the-art model training techniques, there is a research gap on the challenges of developing real-world machine learning systems. For example, our work shows that it is important to move from modelcentric AI towards data-centric $\mathbf{A} \mathbf{I}^{10}$. Practitioners spend most of their time collecting and understanding data, rather than training the model per se. Ultimately, the success of a model stems from the quality of the training and test data, which is where practitioners spend most of their efforts. Moreover, to the best of our knowledge, there is no research literature addressing the challenges of auditing and approving AI software libraries in large-scale organizations.

More research should focus on assisting model governance to reduce bottlenecks in the development process and help ensure that Machine Learning models comply with regulations. Ongoing work argues that model governance literature for fintech is wide and lacks a coherent research agenda (Kavuri and Milne 2019). Yet, related literature suggests that the problem ought to be addressed not only by the fintech industry, but also from the perspective of regulators who have to adapt (Brummer and Yadav 2018; Van Loo 2018).

\subsubsection{Implications for Tool Developers}

Although a number of tools are emerging to aid ML engineering, these solutions fail to address the singularities of different projects. Thus, practitioners are adopting their own customized solutions. For example, spreadsheets are still being used to manually log experiments regardless of the existing automated solutions, such as MLFlow, DVC, Replicate, and so on. It is important to understand what is missing in the current solutions and how we can propose a solution that effectively solves version control to keep track of changes in data, changes in scoring metrics, and executions of different experiments.

Software testing needs to be extended and adapted for Machine Learning software to help effectively test the Machine Learning pipeline at software-, data-, and model-level. It is also necessary to create holistic monitoring solutions that can scale to different models in an organization. There is a need for strategies to help practitioners select the right set of model scoring metrics. Finally, agile development practices are perceived as beneficial but need to be adjusted for AI projects.

\footnotetext{
${ }^{10}$ Andrew Ng nicely explains the importance of data-centric AI in his webinar "A Chat with Andrew on MLOps: From Model-centric to Data-centric AI". Recording available online, retrieved on July 8, 2021: https://www.youtube.com/watch?v=06-AZXmwHjo
} 


\subsubsection{Implications for Educators}

Education of Machine Learning should focus on the whole lifecycle of Machine Learning development, including exploratory analysis with a focus on statistics, data analysis and data visualization. Moreover, practitioners with background on both data science and software engineering are a valuable resource for organizations. This emphasizes the importance of a transdisciplinary approach to AI education (Wang 2003; Nicolescu and Ertas 2008) and it is congruent with previous work that reports that a Software Engineering mindset brings more awareness on the maintainability and stability of an AI project (Arpteg et al. 2018).

\subsubsection{Implications for Organizations Embracing AI}

The embrace of AI stretches the adequacy of well-established processes at organizations. Multi-disciplinary teams are essential to embrace AI: AI experts have the knowledge to try innovative approaches, but will likely have little expertise to identify business value. Thus, knowledge transfer between stakeholders is challenging and might hinder the motivation of developers. New strategies must be outlined to reduce the amount of effort required to document AI projects. Providing AI training to employees can help enable the transition to AI. Not only it makes discussions and decisions about AI projects more effective, but also it helps to identify business opportunities in areas that have not explored the potential of AI yet. Finally, it may happen that different teams will solve the same problem independently (e.g., experiment logs). Although teams are aware of it, they argue that they do not have enough resources to make their solutions reusable (cf. Section 4.8). Thus, organizations should create task forces to make such tools available to all teams.

\subsection{Threats to Validity}

This subsection describes the threats and limitations of the study design and how these are mitigated. These limitations are categorized into researcher bias, respondent bias, interpretive validity, and generalizability, as reported by Maxwell (1992) and Lincoln and Guba (1985).

\subsubsection{Researcher Bias}

Researcher bias is the threat that the results of the study are influenced by the knowledge and assumptions of the researchers, including the influence of the assumptions of the design, analysis, and sampling strategy.

A threat is introduced by the fact that participants are self-selected. This means that there might be employees in the company which should be included in the study but are not selected. During the planning phase, participants are selected with different roles and from different departments to have an as diverse starting point as possible. Thereafter, more participants are found by the recommendation of other interviewees and employees until we reach saturation on the information we get from the interviews, i.e. until no new information or viewpoint is gained from new subjects (Strauss and Corbin 1990).

Moreover, we validated the findings of this study by collecting feedback from relevant stakeholders at ING. Our approach was two-fold: 1) invited relevant stakeholders for a 30minute presentation of our results, followed by a Q\&A and discussion session, and 2) we sent out a report via email with the results and analysis provided in this study. 
The presentation counted with around 15 participants that were not part of the case-study interviews. The main point highlighted by participants was the fact that ING is spending a lot of time and resources to improve their machine learning processes. Hence, they expect to mitigate some of the reported challenges in the meantime.

For the email communications, a total of 19 people were addressed, including stakeholders who have participated in the interviews and stakeholders who have not. One recipient, who had participated in the interviews, took the time to thoroughly read the paper. The recipient mentioned to be in agreement with the findings on the paper but raised one concern the fact that there is not yet a standard methodology at ING to develop machine learning systems. Hence, we report our analyses in this paper as critical observations over existing theories, rather than the de facto model used at ING.

\subsubsection{Respondent Bias}

Respondent bias refers to the situation where respondents do not provide honest responses.

The results of the interviews rely on self-reported data. All people tend to judge the past disproportionately positive. This psychological phenomenon is known as rosy retrospection (Mitchell et al. 1997). Furthermore, interviewees who know golden standards from for example literature may tell how things are supposed to be, in contrast with how they are in reality. These biases are mitigated by reassuring interviewees their answers will not be evaluated or judged and by asking them to think about a particular project they have been working on.

A methodological choice which can form a threat to validity is the fact that interviews are recorded. While the participants themselves permit the recording, they might be extra careful in giving risky statements on the record and therefore introduce bias in their answers. This threat is minimized by assuring the recordings themselves will not be published and all results which will be published are first approved by the corporate communication department.

\subsubsection{Interpretive Validity}

Interpretive validity concerns errors caused by wrongly interpreting participants' statements.

The interviews are processed by field-note taking and memoing. The primary threat to valid interpretation is imposing one's own meaning, instead of understanding the viewpoint of the participants and the meanings they attach to their words. To avoid these interpretation errors, the interviewers used open-ended follow-up questions which allowed the participant to elaborate on answers.

\subsubsection{Generalizability}

Generalizability refers to the extent to which one can extend the results to other settings than those directly studied.

This research is conducted in a single organization - a large financial institution. Despite being only one case, we argue that many of the challenges being solved at ING are relatable to organizations embracing AI into their business. However, results may not seem generalizable to companies of much smaller size or different nature. A bank may be prone to more regulations than most companies and is dealing with more sensitive data. Nevertheless, every company has to comply with privacy regulations like the European GDPR. This 
suggests that results influenced by more strict regulations and compliance are just as relatable to other industries. Multiple case studies at organizations of different scale and nature are required for establishing more general results.

\section{Conclusions}

The goal of this study is to understand the evolution of Machine Learning development and how state-of-the-art lifecycle models fit the current needs of the AI industry.

To that end, we conducted a case study with seventeen Machine Learning practitioners at the fintech company ING.

Our key findings show that traditional Machine Learning lifecycle models are missing essential steps, such as feasibility study, documentation, model evaluation, and model monitoring. This calls for more research to aid practitioners in these essential stages.

We also observe that model governance and technology access are key challenges to the fintech industries leading the AI revolution. Finally, we have found that existing tools to aid Machine Learning development do not address the specificities of different projects, and thus, are seldom adopted by teams.

Our research helps practitioners fine-tune their approach to Machine Learning development to fit fintech use cases. Additionally, it guides educators in defining learning objectives that meet the current needs in the industry.

Finally, this work paves the way for the next research steps in reducing bottlenecks in the Machine Learning lifecycle. In particular, it highlights the need for tool support for exploratory data analysis and data integration techniques, documentation, model governance, monitoring, and version control.

Acknowledgments The authors would like to thank Irene Wijk, Shiler Khedri, and Elvan Kula for their willing contributions to this project. The authors would also like to thank all the participants of the interviews at ING.

Open Access This article is licensed under a Creative Commons Attribution 4.0 International License, which permits use, sharing, adaptation, distribution and reproduction in any medium or format, as long as you give appropriate credit to the original author(s) and the source, provide a link to the Creative Commons licence, and indicate if changes were made. The images or other third party material in this article are included in the article's Creative Commons licence, unless indicated otherwise in a credit line to the material. If material is not included in the article's Creative Commons licence and your intended use is not permitted by statutory regulation or exceeds the permitted use, you will need to obtain permission directly from the copyright holder. To view a copy of this licence, visit http://creativecommons.org/licenses/by/4.0/.

\section{References}

Ahmed Z, Amizadeh S, Bilenko M, Carr R, Chin WS, Dekel Y, Dupre X, Eksarevskiy V, Filipi S, Finley T et al (2019) Machine learning at Microsoft with ML. NET. In: Proceedings of the 25th ACM SIGKDD international conference on knowledge discovery \& data mining. pp 2448-2458

Akkiraju R, Sinha V, Xu A, Mahmud J, Gundecha P, Liu Z, Liu X, Schumacher J (2020) Characterizing machine learning processes: A maturity framework. In: International conference on business process management, Springer, pp 17-31 
Amershi S, Begel A, Bird C, DeLine R, Gall H, Kamar E, Nagappan N, Nushi B, Zimmermann T (2019) Software engineering for machine learning: a case study. In: Proceedings of the 41st International conference on software engineering, software engineering in practice. IEEE Press, pp 291-300

Arpteg A, Brinne B, Crnkovic-Friis L, Bosch J (2018) Software engineering challenges of deep learning, IEEE

Bernardi L, Mavridis T, Estevez P (2019) 150 successful machine learning models: 6 lessons learned at Booking.com. In: Proceedings of the 25th ACM SIGKDD international conference on knowledge discovery \& data mining. ACM, pp 1743-1751

Breck E, Cai S, Nielsen E, Salib M, Sculley D (2017) The ml test score: A rubric for ml production readiness and technical debt reduction, IEEE

Brereton P, Kitchenham BA, Budgen D, Li Z (2008) Using a protocol template for case study planning. In: EASE, Citeseer, vol 8, pp 41-48

Brummer C, Yadav Y (2018) Fintech and the innovation trilemma. Geo LJ 107:235

Cruz L, Abreu R (2019) Catalog of energy patterns for mobile applications. Empir Softw Eng. https://doi. org/10.1007/s10664-019-09682-0

Damiani E, Frati F (2018) Towards conceptual models for machine learning computations. In: International conference on conceptual modeling. Springer, pp 3-9

Ericson G, Rohm WA, Martens J, Sharkey K, Casey C, Harvey B, Schonning N (2017) Team data science process documentation. Retrieved September $2020 \mathrm{https} / / /$ docs.microsoft.com/en-us/azure/ machine-learning/team-data-science-process/

Fasick FA (1977) Some uses of untranscribed tape recordings in survey research. Public Opinion Q 41(4):549-552

Fereday J, Muir-Cochrane E (2006) Demonstrating rigor using thematic analysis: A hybrid approach of inductive and deductive coding and theme development. Int J Qual Methods 5(1):80-92

Haakman M, Cruz L, Huijgens H, van Deursen A (2020) Machine learning behind the scenes: An exploratory study in fintech - case study protocol. https://1drv.ms/b/s!AuvX_CBP4YARcBBYdYAOG8qLGIk

Halcomb EJ, Davidson PM (2006) Is verbatim transcription of interview data always necessary? Appl Nurs Res 19(1):38-42

Hill C, Bellamy R, Erickson T, Burnett M (2016) Trials and tribulations of developers of intelligent systems: A field study. In: 2016 IEEE symposium on visual languages and human-centric computing (VL/HCC). IEEE, pp 162-170

ING (2019) ING at a glance. https://www.ing.com/About-us/Profile/ING-at-a-glance.htm

15288 ISO/IEC/IEEE (2015) Systems and software engineering - System life cycle processes. Institute of Electrical and Electronic Engineers (IEEE), New York

Kavuri AS, Milne A (2019) Fintech and the future of financial services: What are the research gaps CAMA Working Paper No 18/2019

Kim M, Zimmermann T, DeLine R, Begel A (2017) Data scientists in software teams: State of the art and challenges. IEEE Trans. Softw. Eng. 44(11):1024-1038

Larios Vargas E, Aniche M, Treude C, Bruntink M, Gousios G (2020) Selecting third-party libraries: The practitioners' perspective. In: Proceedings of the 28th ACM joint meeting on european software engineering conference and symposium on the foundations of software engineering, pp 245-256

Lin J, Ryaboy D (2013) Scaling big data mining infrastructure: the Twitter experience. Acm SIGKDD Explorations Newsletter 14(2):6-19

Lincoln Y, Guba E (1985) Naturalistic inquiry. SAGE, Newbury Park

Lwakatare LE, Crnkovic I, Rånge E, Bosch J (2020) From a data science driven process to a continuous delivery process for machine learning systems. In: International conference on product-focused software process improvement. Springer, pp 185-201

Mariscal G, Marban O, Fernandez C (2010) A survey of data mining and knowledge discovery process models and methodologies. Knowl Eng Rev 25(2):137-166

Martínez-Plumed F, Contreras-Ochando L, Ferri C, Flach P, Hernández-Orallo J, Kull M, Lachiche N, Ramírez-Quintana MJ (2017) Casp-dm: Context aware standard process for data mining. arXiv: 1709.09003

Martínez-Plumed F, Contreras-Ochando L, Ferri C, Orallo JH, Kull M, Lachiche N, Quintana MJR, Flach PA (2019) CRISP-DM twenty years later: From data mining processes to data science trajectories. IEEE Trans Knowl Data Eng

Maxwell J (1992) Understanding and validity in qualitative research. Harvard Educ Rev 62(3):279-301

Mead C, Ismail M (1989) Analog VLSI implementation of neural systems. Springer, Berlin

Menzies T (2019) The five laws of se for ai. IEEE Softw. 37(1):81-85 
Mitchell M, Wu S, Zaldivar A, Barnes P, Vasserman L, Hutchinson B, Spitzer E, Raji ID, Gebru T (2019) Model cards for model reporting. In: Proceedings of the conference on fairness, accountability, and transparency, pp 220-229

Mitchell TR, Thompson L, Peterson E, Cronk R (1997) Temporal adjustments in the evaluation of events: The "rosy view". J Exper Soc Psychol 33(4):421-448

Moyle S, Jorge A (2001) Ramsys-a methodology for supporting rapid remote collaborative data mining projects, vol 64

Nicolescu B, Ertas A (2008) Transdisciplinary theory and practice. USA, TheATLAS

Rollins J (2015) Foundational methodology for data science. Domino Data Lab, Inc. Whitepaper

Ryan MJ, Wheatcraft LS (2017) On the use of the terms verification and validation. In: INCOSE International Symposium, vol 27, pp 1277-1290. https://doi.org/10.1002/j.2334-5837.2017.00427.x

Sculley D, Holt G, Golovin D, Davydov E, Phillips T, Ebner D, Chaudhary V, Young M, Crespo JF, Dennison D (2015) Hidden technical debt in machine learning systems. In: Advances in neural information processing systems, pp 2503-2511

Serban A, van der Blom K, Hoos H, Visser J (2020) Adoption and effects of software engineering best practices in machine learning. In: Empirical software engineering and measurement

Shearer C (2000) The CRISP-DM model: the new blueprint for data mining. J Data Warehous 5(4):13-22

Stol KJ, Ralph P, Fitzgerald B (2016) Grounded theory in software engineering research: a critical review and guidelines. In: Proceedings of the 38th International conference on software engineering, pp 120-131

Strauss A, Corbin J (1990) Basics of qualitative research. Sage publications, Thousand Oaks

Van Loo R (2018) Making innovation more competitive: the case of fintech. UCLA L Rev 65:232

Wang Y (2003) Cognitive informatics: A new transdisciplinary research field. Brain and Mind 4(2):115-127. https://doi.org/10.1023/A:1025419826662

Wengraf T (2001) Qualitative research interviewing: Biographic narrative and semi-structured methods. SAGE, Newbury Park

Wu CJ, Brooks D, Chen K, Chen D, Choudhury S, Dukhan M, Hazelwood K, Isaac E, Jia Y, Jia B et al (2019) Machine learning at facebook: Understanding inference at the edge, IEEE

Wu X, Zhu X, Wu GQ, Ding W (2013) Data mining with big data. IEEE Trans Knowl Data Eng 26(1):97-107

Yin RK (2017) Case study research and applications: Design and methods. Sage publications, Thousand Oaks

Zhang JM, Harman M, Ma L (2020) Liu Y, Machine learning testing, Survey, landscapes and horizons. IEEE Trans Softw Eng

Publisher's note Springer Nature remains neutral with regard to jurisdictional claims in published maps and institutional affiliations. 